\title{
Patient repositioning and the amelioration of airway obstruction by an anterior mediastinal tumor during general anesthesia
} -A case report-

\author{
Won Joon Choi, Yun Hong Kim, Jeong Min Mok, Soo Il Choi, and Hyun Soo Kim \\ Department of Anesthesiology and Pain Medicine, Kangbuk Samsung Hospital, Sungkyunkwan University School of Medicine, Seoul, \\ Korea
}

An 18-year-old male with huge anterior mediastinum mass was scheduled for thoracotomic incisional biopsy under general anesthesia after failed fluoroscopy-guided percutaneous needle biopsy. Under propofol and succinylcholine anesthesia, intubation was successfully achieved using a Univent tube. However, when we changed the patient's position from supine to right lateral decubitus, oxygen saturation declined. He was then positioned supine, but hypoxemia did not improve. Because the tumor expanded toward the left thoracic field, we considered that the left lateral decubitus position might help relieve the mass effect on the main bronchus. His position was changed accordingly and soon after, hypoxemia improved and surgery was undertaken under cardiopulmonary bypass (CPB). The biopsy was successfully performed under CPB without complication. (Korean J Anesthesiol 2010; 59: 206-209)

Key Words: Airway obstruction, General anesthesia, Mediastinum, Posture, Resuscitation, Tumor.

A mass located in anterior mediastinum can cause airway obstruction during general anesthesia [1-5], and the majority of severe perioperative adverse complications related to anterior mediastinal masses have been reported in patients under 18 years old [6]. Accordingly, local anesthesia for the surgery of an anterior mediastinal mass is preferred in young adults. However, if surgery must be performed under general anesthesia, operators should be aware of this potentially fatal complication associated with a mediastinal mass. We report a case of unexpected airway obstruction caused by an anterior mediastinal mass during general anesthesia. The patient was successfully resuscitated by repositioning, and a thoracotomic incisional biopsy was then performed under cardiopulmonary bypass; the patient subsequently recovered without any major complication. We also include a review of the literature regarding the effects of positional changes on cardiorespiratory complications caused by an anterior mediastinal mass.

Received: October 6, 2009. Revised: 1st, October 28, 2009; 2nd, November 13, 2009. Accepted: November 26, 2009.

Corresponding author: Yun Hong Kim, M.D., Department of Anesthesiology and Pain Medicine, Kangbuk Samsung Hospital, Sungkyunkwan University School of Medicine, 108, Pyeong-dong, Jongno-gu, Seoul 110-746, Korea. Tel: 82-2-2001-2322, Fax: 82-2-2001-2326, E-mail: yhkim12@yahoo.co.kr

(c) This is an open-access article distributed under the terms of the Creative Commons Attribution Non-Commercial License (http:// creativecommons.org/licenses/by-nc/3.0/), which permits unrestricted non-commercial use, distribution, and reproduction in any medium, provided the original work is properly cited. 


\section{Case Report}

An 18-year-old male presented with a complaint of chest discomfort, exertional dyspnea, and an intractable dry cough. Chest radiography revealed a mass like lesion in left upper mediastinum and trachea deviated to the right-side. Multi detector computed tomography (MDCT) revealed a $12 \times 9$ $\times 15 \mathrm{~cm}$ sized heterogenously enhancing mass in anterior mediastinum extending ventrally into the left pectoralis major muscle, and dorsally into the aortic arch, pulmonary artery trunk, and descending aorta, and encroaching the left lung field (Fig. 1 and 2). However, the diameters of involved vessels were

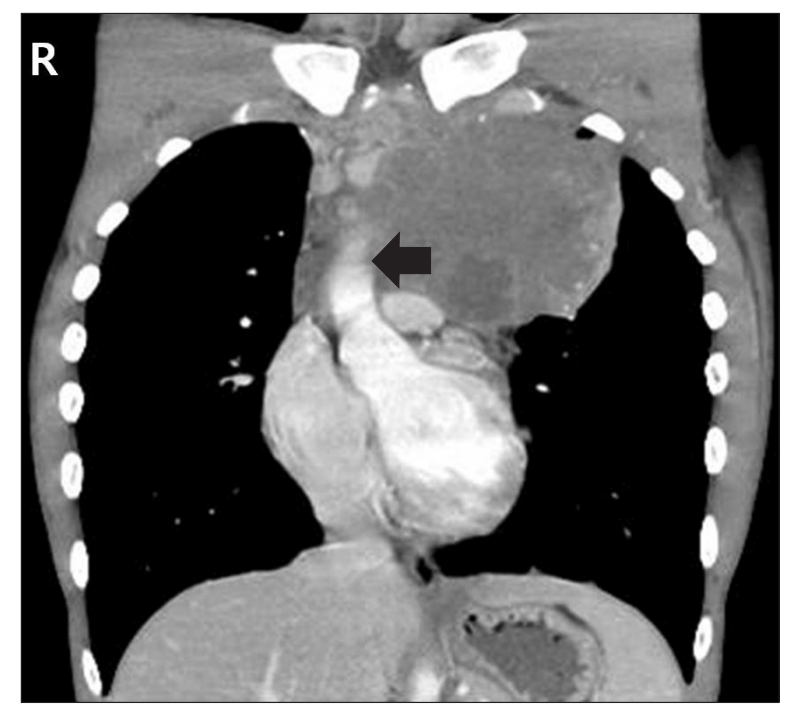

Fig. 1. Preoperative coronal contrast-enhanced reformatted multi detector computed tomography image showing the mass located above the cardiac chamber wrapping the ascending aorta (black arrow). The tumor mass leant toward the left lung field.

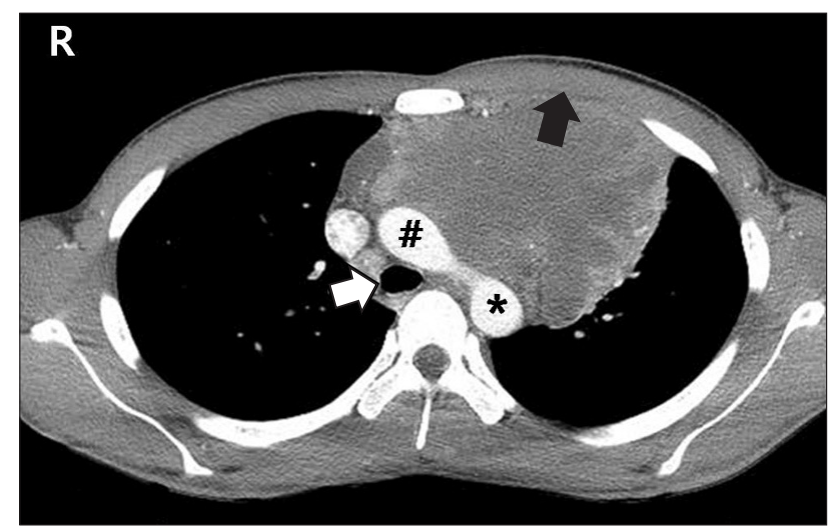

Fig. 2. Preoperative transverse reformatted multi detector computed tomography image revealing that a patent tracheal cross-section (white arrow). The mass encroached the left pectoralis major muscle (black arrow), wrapped the ascending (\#) and descending aorta (*), and expanded into the left lung field. preserved (not shown), and the trachea and both main bronchi were patent (Fig. 2 and 3). Transthoracic echocardiography demonstrated minimal pericardial effusion with moderate mitral regurgitation and a left ventricular ejection fraction of 52\%. Pulmonary function test (PFT) findings were within normal limits, and including a peak expiratory flow rate (PEFR) of $50 \%$ the predicted value.

Although the patient underwent a fluoroscopy-guided percutaneous needle lung biopsy twice, the type of tumor was not confirmed, and thus, a thoracotomic incisional biopsy was decided upon under general anesthesia. After intravenously injecting $1 \%$ propofol and succinylcholine in the supine position, a Univent tube was successfully intubated by direct laryngoscopy. Anesthetic maintenance was then carried out by the target-controlled infusion of propofol and remifentanil with $\mathrm{O}_{2}$, air, and intermittent intravenous rocuronium. During the first 45 minutes after induction in the supine position, end-tidal carbon dioxide partial pressure $\left(\mathrm{PetCO}_{2}\right)$, heart rate (HR) and systolic/diastolic blood pressures were 37-39 $\mathrm{mmHg}, 109-$ $120 \mathrm{bpm}$, and 121-181/85-103 mmHg, respectively. Even after closing the left main bronchus using a bronchial blocker, pulse oximetry $\mathrm{O}_{2}$ saturation $\left(\mathrm{SpO}_{2}\right)$ was maintained at $97-99 \%$, and central venous pressure (CVP) was maintained at $14-15 \mathrm{mmHg}$ under controlled positive pressure ventilation. He was then placed in the right lateral decubitus for the left thoracotomy approach, and for the first 30 minutes after this positional change, his vital signs remained unchanged. However, $\mathrm{SpO}_{2}$ then began to decline, and fiberoptic bronchoscopy demonstrated the left main bronchus was almost totally obstructed, and that the ballooned bronchial blocker had been

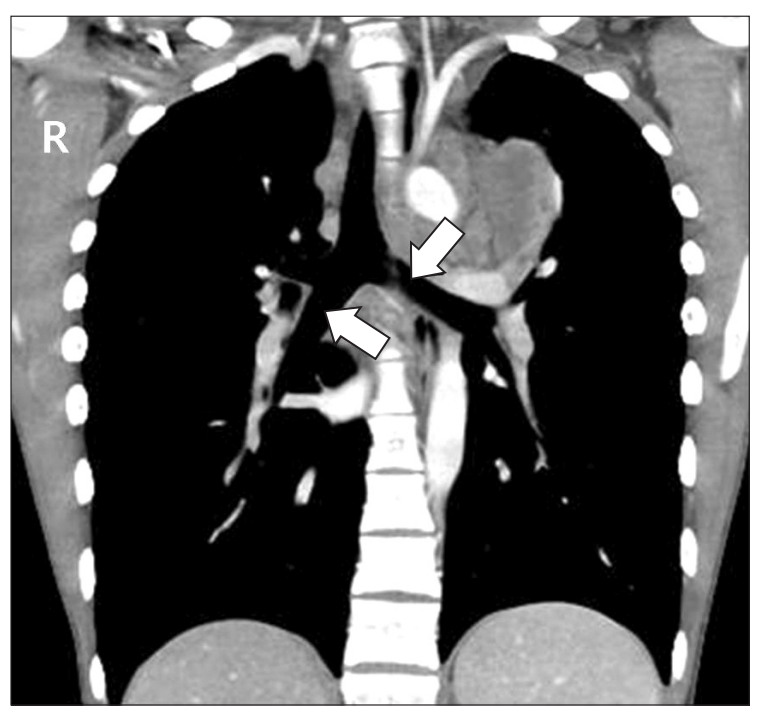

Fig. 3. Preoperative coronal reformatted multi detector computed tomography image showing patency of the trachea and both main bronchi (white arrows). 
dislodged into the carina. Accordingly, we immediately turned patient supine to relieve the mass effect on the main bronchi. However, hypoxemia was further aggravated up to $65 \%$ even after two lung ventilation and increasing the inspiratory oxygen fraction $\left(\mathrm{FiO}_{2}\right)$ to 1.0. The sitting position also failed to improve oxygenation. Furthermore, CVP increased gradually from 17 to $65 \mathrm{mmHg}$ after the $\mathrm{SpO}_{2}$ decreased. Nevertheless, blood pressure $(112-123 / 72-77 \mathrm{mmHg})$ and $\mathrm{PetCO}_{2}(32-36 \mathrm{mmHg})$ remained in their normal ranges.

Eventually, the patient was placed in the left lateral decubitus position to detach the mass from the site of obstruction by gravity, and thereafter, $\mathrm{SpO}_{2}$ improved from $79 \%$ to 93 and then to $97-99 \%$, and CVP simultaneously decreased from 65 to $18-20$ mmHg. Surgery was then undertaken under a partial (femorofemoral) cardiopulmonary bypass (CPB) in the right lateral decubitus position. The total bypass time was 150 minutes, and blood pressures and heart rates perioperatively were 89120/44-50 mmHg and 95-115 bpm, respectively. During bypass, core temperature was maintained at normothermia $\left(36^{\circ} \mathrm{C}\right)$ and $\mathrm{PetCO}_{2}$ was $36-43 \mathrm{mmHg}$. A frozen biopsy later confirmed Hodgkin's lymphoma. After weaning the patient off $\mathrm{CPB}$, his $\mathrm{SpO}_{2}$ was $100 \%$, PetCO $242-46 \mathrm{mmHg}$, and CVP 12-16 mmHg under controlled positive pressure ventilation supplying $\mathrm{FiO}_{2}$ 1.0. Muscle relaxation was reversed using glycopyrrolate and pyridostigmine. After observing full recovery of spontaneous respiration and of extremity muscle power, we extubated the Univent tube and transferred the patient to an intensive care unit (ICU) with $\mathrm{O}_{2} 5 \mathrm{~L} / \mathrm{min}$ administered through a venturi mask. In the ICU, no signs of hypoxemia were evident $\left(\mathrm{SpO}_{2} 98 \%\right)$, and no cardiorespiratory or neurologic abnormalities were found. On postoperative day 2 , the patient was moved to a general hospital ward.

\section{Discussion}

General anesthesia can have hazardous effects on the airways and on the cardiovascular system. Loss of muscle tone, especially that induced by muscle relaxants, makes airways susceptible to collapse due to external compression by mediastinal masses [4], and furthermore, the supine position leads to cephalic displacement of the diaphragm and reduces thoracic anteroposterior diameter. In addition, controlled positive pressure ventilation reduces negative intrathroacic pressure, which tend to move the mediastinal mass away from the airway and cardiovascular system. Furthermore, in combination, these influences can provoke severe airway obstruction in the supine position under general anesthesia, and thus, the use of muscle relaxants is not recommended under general anesthesia $[1,7]$, and accordingly, surgery is best performed under local anesthesia [1].
However, when surgery must be performed under general anesthesia, especially with muscle relaxants, resuscitating methods should be provided to manage cardiorespiratory complications. In addition, a number of resuscitating methods have been introduced, such as, positional changes, tracheobronchial stenting, and rigid bronchoscopy to relieve airway obstructions and cardiopulmonary bypass [6-10].

Changing posture is more feasible than any other method, and we recommend before induction that the position which frees the patients from tumor-related cardiorespiratory complications be determined $[7,8,11]$. This 'comfortable' position can then be used during general anesthesia.

However, if this comfortable position is not determined preoperatively, the sitting position is preferable for resuscitation in the presence of an airway obstruction [10]. This position moves the diaphragm caudally and alleviates the pressure of the mediastinal mass on surrounding organs [12]. However, as in our case, the sitting position does not always effectively relieve cardiorespiratory complications during general anesthesia [4], and as has been previously reported, the lateral decubitus or prone position should then be adopted to enable the nature of the complication to be determined [10]. Nevertheless, it is unclear which position is best for clearing an airway obstruction due to a mediastinal mass during general anesthesia $[4,5,12,13]$. The right lateral decubitus position could either prevent compression of the cardiorespiratory system during induction or relieve hypoxemia and hypotension during general anesthesia, irrespective of whether a tumor in the anterior mediastinum primarily expands toward the left or right side of the chest $[5,12,13]$. However, in another report, the right lateral decubitus position did not solve the hypoxemia or hypotension caused by an anterior mediastinal mass, which was misdiagnosed as a pericardial effusion by preoperative echocardiography [4].

In addition to changing posture, emergency percutaneous cardiopulmonary bypass support $[9,11]$ or extracorporeal membrane oxygenation (ECMO) support [14] can be applied to an unexpected airway collapse during general anesthesia caused by a mediastinal mass. Furthermore, when a patient with a mediastinal mass complains of cardiorespiratory symptoms before induction, it may be advisable to have a prepared cardiopulmonary support system available in the operating room.

Our case showed a rise in CVP after being positioned in the right lateral decubitus that was sustained even in the sitting position. The right heart and pulmonary artery, which are at lower pressures than the left heart and aorta, are more vulnerable to compression by a mediastinal mass [6]. Although the pulmonary trunk was not compressed by preoperative MDCT in our case, the positional change might have caused 
a mass effect on the right heart or pulmonary artery and the observed increase in CVP. However, the maintenance of normal blood pressures suggested a partial obstruction of the pulmonary circulation [9]. Furthermore, the airway below the right main bronchus was probably only partially collapsed because $\mathrm{PetCO}_{2}$ remained in the normal range.

It is important that complications during anesthesia be predicted preoperatively. When the cross-sectional area of the trachea is compressed by more than $50 \%$ of predicted, or pericardial or pleural effusions are visualized by preoperative $\mathrm{CT}$, the patient may be more vulnerable to perioperative complications $[1,15]$. Furthermore, a peak expiratory flow rate (PEFR) by the pulmonary function test (PFT) of less than $50 \%$ of the predicted value should raise concerns about complications due to a mediastinal mass [1].

In our patient, the preoperative tracheal cross-sectional area was patent, but PEFR by PFT was $50 \%$ of predicted and minimal pericardial effusion was observed by echocardiography. Our patient also complained of exertional dyspnea, and thus, we should have expected hypoxemia or hypotension during general anesthesia.

In conclusion, positional changes can be used to relieve an unexpected airway obstruction caused by an anterior mediastinal mass. However, before applying this method, it should be considered whether the intended posture is likely to be hazardous or effective. We recommend that when a patient is positioned that the center of the mass should be located in the dependent site of the body.

\section{References}

1. Perger L, Lee EY, Shamberger RC. Management of children and adolescents with a critical airway due to compression by an anterior mediastinal mass. J Pediatr Surg 2008; 43: 1990-7.

2. Akhtar TM, Ridley S, Best CJ. Unusual presentation of acute upper airway obstruction caused by an anterior mediastinal mass. Br J Anaesth 1991; 67: 632-4.

3. Szokol JW, Alspach D, Mehta MK, Parilla BV, Liptay MJ. Intermittent airway obstruction and superior vena cava syndrome in a patient with an undiagnosed mediastinal mass after cesarean delivery. Anesth Analg 2003; 97: 883-4.

4. Lin CM, Hsu JC. Anterior mediastinal tumour identified by intraoperative transesophageal echocardiography. Can J Anaesth 2001; 48: 78-80.

5. Lee JH, Lim HS, Nam KH, Lee KN, Moon JI. Superior vena caval and airway obstruction on induction of anesthesia in a patient with anterior mediastinal mass: a case report. Korean J Anesthesiol 1999; 37: 153-8.

6. Narang S, Harte BH, Body SC. Anesthesia for patients with a mediastinal mass. Anesthesiol Clin North America 2001; 19: 559-79.

7. Erdös G, Tzanova I. Perioperative anaesthetic management of mediastinal mass in adults. Eur J Anaesthesiol 2009; 26: 627-32.

8. Slinger P, Karsli C. Management of the patient with a large anterior mediastinal mass: recurring myths. Curr Opin Anaesthesiol 2007; 20: 1-3.

9. Takeda S, Miyoshi S, Omori K, Okumura M, Matsuda H. Surgical rescue for life-threatening hypoxemia caused by a mediastinal tumor. Ann Thorac Surg 1999; 68: 2324-6.

10. Gothard JW. Anesthetic considerations for patients with anterior mediastinal masses. Anesthesiol Clin 2008; 26: 305-14.

11. Asai T. Emergency cardiopulmonary bypass in a patient with a mediastinal mass. Anaesthesia 2007; 62: 859-60.

12. Hammer GB. Anaesthetic management for the child with a mediastinal mass. Paediatr Anaesth 2004; 14: 95-7.

13. Khanna S, Varghese S, Vaijyanath P, Arora K, Aggarwal A. Giant anterior mediastinal mass causing airway obstruction. Ann Card Anaesth 2005; 8: 169.

14. Chao VT, Lim DW, Tao M, Thirugnanam A, Koong HN, Lim CH. Tracheobronchial obstruction as a result of mediastinal mass. Asian Cardiovasc Thorac Ann 2006; 14: e17-8.

15. Béchard P, Létourneau L, Lacasse Y, Côté D, Bussières JS. Perioperative cardiorespiratory complications in adults with mediastinal mass: incidence and risk factors. Anesthesiology 2004; 100: 826-34. 